\title{
Psychometric Properties of the Korean Translation of the Attention-Deficit/Hyperactivity Disorder Stigma Questionnaire
}

\author{
Soo Jung Rim, Hyesue Jang, and Subin Park \\ Department of Research Planning, Mental Health Research Institute, National Center for Mental Health, Seoul, Korea
}

\begin{abstract}
Objectives: This study evaluated the psychometric properties of the Korean version of the attention-deficit/hyperactivity disorder (ADHD) Stigma Questionnaire (ASQ) and the effect of the source of information about mental health on ADHD stigma.

Methods: The Korean translation of the ASQ was prepared, and 673 participants, 20-64 years of age, completed the questionnaire using an online panel survey in South Korea. The participants also completed questionnaires detailing sociodemographic variables and the source of their mental health knowledge. Cronbach's alpha coefficient was used to explore the internal consistency of the ASQ. Factor analysis using Varimax rotation was conducted to investigate the structure of the ASQ.

Results: The 26-item ASQ demonstrated excellent internal consistency (Cronbach's alpha=0.940). Factor analysis supported a threefactor structure, including Concerns with Public Attitudes, Negative Self-Image, and Disclosure Concerns. There were no significant differences in the total ASQ scores according to sociodemographic characteristics. Participants who reported the internet as their major source of information about mental health showed higher ASQ scores compared to those who used other sources for mental health information.
\end{abstract}

Conclusion: The Korean translation of the ASQ has acceptable psychometric properties among Korean adults. Inaccurate information from the internet could increase the stigma toward ADHD.

Key Words: Attention-deficit/hyperactivity disorder; Stigma; Attention-deficit/hyperactivity disorder Stigma Questionnaire.

Received: December 27, 2017 / Revision: March 22, 2018 / Accepted: March 28, 2018

Address for correspondence: Subin Park, Department of Research Planning, Mental Health Research Institute, National Center for Mental Health, 127 Yongmasan-ro, Gwangin-gu, Seoul 04933, Korea

Tel: +82-2-2204-0108, Fax: +82-2-2204-0280, E-mail: subin-21@hanmail.net

\section{INTRODUCTION}

Attention-deficit/hyperactivity disorder (ADHD) is a common mental disorder in children and adults. ${ }^{1)}$ The prevalence of ADHD varies significantly around the world. A meta-analysis reported the worldwide prevalence of ADHD to be $5.29 \% .{ }^{2)}$ Globally, childhood ADHD persists into adulthood in approximately $50 \%$ of all cases. ${ }^{3)}$

Stigma is defined as stereotyped beliefs, prejudiced attitudes, and discriminatory behaviors against particular groups. ${ }^{4)}$ Stigmatization appears globally and is evident in many forms: public stigma, self-stigma, and courtesy stigma. ${ }^{5-7)}$ Public stigma occurs when a large group of people agrees on negative ideas or attitudes about a particular group. As a consequence of public stigma, self-stigma could occur. Self-stigma arises when an individual who is experiencing stigma gen-

This is an Open Access article distributed under the terms of the Creative Commons Attribution Non-Commercial License (http://creativecommons.org/licenses/by-nc/4.0) which permits unrestricted non-commercial use, distribution, and reproduction in any medium, provided the original work is properly cited. erates a degraded identity of self. ${ }^{6}$ Moreover, people related to individuals experiencing stigma may also be stigmatized (i.e., courtesy stigma). ${ }^{7}$

Several experimental studies have presented evidence of stigmatization towards individuals with ADHD. For example, individuals with ADHD are perceived as less socially desirable compared to individuals with general medical problems or individuals with an ambiguous weakness, for instance, someone who is a perfectionist. ${ }^{8)}$ Moreover, one study ${ }^{9)}$ reported that approximately $20 \%$ of participants indicated they would be 'probably' or 'definitely' unwilling to interact with children that have ADHD.

As a result of stigma, individuals with ADHD can be socially rejected ${ }^{7)}$ and may have restricted life opportunities and suboptimal social interactions. ${ }^{10,11)}$ For example, Hoza et al. ${ }^{12)}$ found that children diagnosed with ADHD were less favored by their peers and friends. Moreover, individuals related to patients with ADHD suffer from stigmatization as well. According to one study, ${ }^{13)}$ parents of children with 
ADHD are blamed for their child's characteristics that do not fit into social norms. This leads to an increased risk for depression and anxiety in mothers of children with ADHD. ${ }^{14)}$

As a consequence, stigmatization of people with mental disorders is a major concern in various disciplines, including clinical psychology, psychiatry, and public health. ${ }^{15)}$ Accordingly, in the past decade, various studies have addressed the effect of stigma, prejudice, and discrimination against individuals with mental disorders. ${ }^{16)}$ Despite these efforts, stigmatization towards such individuals has not decreased. ${ }^{17)}$ The majority of these studies have focused on depression, schizophrenia, and bipolar disorder ${ }^{18-22)}$; however, the stereotypical content of one mental disorder cannot be generalized to all mental disorders. ${ }^{23)}$ Therefore, more studies should focus on stigma toward under-investigated mental disorders.

When studying stigma towards mental health, cultural aspects need to be considered, because cultural aspects influence attitudes toward mental illness. ${ }^{24)}$ In Asian cultures, there is evident public stigma towards mental illness because it is regarded as a disgrace on one's family lineage. ${ }^{24)}$ Specifically in South Korea, mental illness is shameful and is considered to be a result of an ancestral spirit haunting the patient as a punishment for their past wrong-doing, or as a result of misfortune. ${ }^{25)}$ However, there are very few studies on assessing or reducing stigma towards mental illness in South Korea. ${ }^{26)}$

Despite the evident stigma towards ADHD, this topic has been under-investigated. ${ }^{7)}$ Specifically, no measurements had been developed to assess stigma against ADHD; finally, the ADHD Stigma Questionnaire (ASQ) was developed. ${ }^{27)}$ The ASQ measures stigma using three factors: Disclosure Concerns, Negative Self-Image, and Concern with Public Attitudes. Since stigmatization of mental illness is culturally formed, ${ }^{5)}$ it is important to evaluate whether it is appropriate to use the ASQ in each country when assessing stigma towards ADHD.

Moreover, the perception of the general public towards mental health is influenced by how the mass media portrays individuals with mental disorders. ${ }^{28)}$ According to Klin and Lemish, ${ }^{29)}$ information and descriptions of mental disorders in the media are often inaccurate and exaggerated, and go as far as to describe individuals with mental disorders as dangerous. For intervention purposes, it is important to determine which sources of information affect the perception of the general population toward mental disorders.

This study evaluated the psychometric properties, including internal consistency and factor structure, of the Korean translation of the ASQ. Additionally, we examined the correlates of the stigmatization of ADHD (sociodemographic variables, having a mental disorder, having a friend or relative with ADHD, having a friend or relative with any mental disorders, and the sources where individuals acquire infor- mation about mental health).

\section{METHODS}

\section{Participants}

A sample of 632 Korean adults participated in an online panel survey in September 2017. Recruitment was coordinated by an online research service that operates its own consumer panel site. Korean adults aged 20 to 59 years were included in the study through random sampling by utilizing the proportionate allocation of the age distribution in South Korea. Email invitations were randomly sent to potential respondents who had completed a survey at least once within the prior 12 months from the consumer panel site (www.invight.co.kr) operated by the research service. Information for the study was found on the online survey site. Participants read the instructions and, if they agreed to participate, clicked the 'participate' button. This study was reviewed and approved by the Institutional Review Board of the National Center for Mental Health (IRB No. 116271-2017-38).

\section{Materials}

\section{ADHD Stigma Questionnaire (ASQ)}

The ASQ developed by Kellison et al. ${ }^{27)}$ is an adaptation of the 40-item Human Immunodeficiency Virus (HIV) Stigma Scale. ${ }^{30)}$ The HIV Stigma Scale was only targeted to individuals with HIV; in contrast, the ASQ was intended not only for individuals diagnosed with ADHD but also for teachers and non-clinical populations. Therefore, the items were changed from first person to third person. Additionally, all items were re-worded to measure stigma towards ADHD. The items were reviewed by various experts in psychiatry, clinical psychology, social work, education, school psychology, counselling, and statistics. ${ }^{27)}$ After the review process, some items that had poor conceptual fit with ADHD were eliminated (these two items focused on infectious etiology). Also, an additional eight items were eliminated because they had low item-total correlations in total and in subscale analyses. As a result, the ASQ was comprised of 26 items focused on one of three factors regarding stigma towards ADHD: Disclosure Concerns, Negative Self-Image, and Concern with Public Attitudes. Each item was rated on a 4-point Likert scale (1: strongly disagree, 2: disagree, 3: agree, and 4: strongly agree).

For the purpose of our study, the ASQ was translated to Korean by a bilingual (Korean and English) individual (H.J.) who holds a master's degree in clinical psychology. Back translation was completed by an English editing service (H. Company) by a native English speaker. 


\section{Sociodemographic data}

Information on age, sex, socioeconomic status, education level, and religion was collected. Additionally, the participants were asked whether they had a family member/relative/friend with a current or previous diagnosis of ADHD or any other mental disorders. The participants were also asked whether they had been diagnosed with any mental disorders.

\section{Sources of information about mental health}

Sources that participants used to gather information about mental health was evaluated by the following question: "Where do you usually acquire information about mental health?" The answer choices were 1) television and newspaper, 2) the internet, 3) books, 4) lecture(s) on mental health, 5) family, friends, and neighbors, and 6) other sources (with the participants providing the sources). Participants were allowed to select multiple responses.

\section{Statistical analyses}

Cronbach's alpha coefficient was used to explore the internal consistency of the ASQ. Factor analyses using Varimax rotation were conducted to investigate the structure of the ASQ. ASQ scores were compared according to sociodemographic characteristics and the sources of information about mental health using t-tests or analyses of variance. All statistical analyses were performed with SPSS 21.0 (IBM Corp., Armonk, NY, USA), with statistical significance defined as $\alpha=0.05$.

\section{RESULTS}

\section{Socio-demographic characteristics of the study population}

A total of 632 participants, aged 20-59 years (mean age, $40.51 \pm 11.31$ years; 313 males, 319 females) responded to the survey.

\section{Internal consistency reliability}

The coefficient alphas for the 26 combined items of the Korean version of the ASQ showed excellent internal consistency (Cronbach's alpha=0.94). As shown in Table 1, none of the items was considered for deletion to increase the internal consistency. Also, correlations were performed to investigate the relationship between each item and the total scale. Item 18 demonstrated the highest correlation compared to all other items (Table 1).

\section{Factor structure}

Table 2 presents the mean scores for each of the 26 items
Table 1. Correlations between each of the 26 items and the total score

\begin{tabular}{|c|c|c|}
\hline & $\begin{array}{c}\text { Corrected item-total } \\
\text { correlation }\end{array}$ & $\begin{array}{l}\text { Cronbach alpha } \\
\text { if item deleted }\end{array}$ \\
\hline 1 & 0.307 & 0.942 \\
\hline 2 & 0.495 & 0.939 \\
\hline 3 & 0.562 & 0.938 \\
\hline 4 & 0.552 & 0.939 \\
\hline 5 & 0.626 & 0.937 \\
\hline 6 & 0.616 & 0.938 \\
\hline 7 & 0.641 & 0.937 \\
\hline 8 & 0.689 & 0.937 \\
\hline 9 & 0.630 & 0.937 \\
\hline 10 & 0.599 & 0.938 \\
\hline 11 & 0.663 & 0.937 \\
\hline 12 & 0.679 & 0.937 \\
\hline 13 & 0.637 & 0.937 \\
\hline 14 & 0.603 & 0.938 \\
\hline 15 & 0.668 & 0.937 \\
\hline 16 & 0.586 & 0.938 \\
\hline 17 & 0.651 & 0.937 \\
\hline 18 & 0.694 & 0.937 \\
\hline 19 & 0.650 & 0.937 \\
\hline 20 & 0.507 & 0.939 \\
\hline 21 & 0.571 & 0.938 \\
\hline 22 & 0.610 & 0.938 \\
\hline 23 & 0.598 & 0.938 \\
\hline 24 & 0.556 & 0.938 \\
\hline 25 & 0.575 & 0.938 \\
\hline 26 & 0.536 & 0.939 \\
\hline
\end{tabular}

and the result of the factor analysis (Varimax rotated). The three factors together accounted for $54.17 \%$ of the variance. All three factors were readily interpretable. The first factor, labeled 'Concern with Public Attitudes' comprised items 10, $12,14,16,20,21,22,25$, and 26 , and accounted for $19.18 \%$ of the variance. The second factor, labeled 'Negative Self-Image' comprised items $1,2,3,4,6,7,8$, 9, and 11, and accounted for $17.73 \%$ of the variance. The last factor, labeled 'Disclosure Concerns' comprised items 5, 13, 15, 17, 18, 19, 23, and 24 , and accounted for $17.26 \%$ of the variance.

\section{ASQ scores according to participants' characteristics}

The mean ASQ score of all participants was 70.90 \pm 10.98 . We investigated the ASQ scores according to sociodemographic characteristics such as sex, age, religion, family income, education level, and participant's diagnosis of any mental disorder, diagnosis of ADHD in family/relatives/friends of participants, and diagnosis of any mental disorders in family, relatives, or friends of participants. There were no statistically significant differences in ASQ scores when analyzed 
Table 2. Means for questionnaire items and factor loadings

\begin{tabular}{|c|c|c|c|}
\hline & Factor/items & Loading & Mean (SD) \\
\hline \multicolumn{3}{|c|}{ Concern with public attitudes } & $24.50(4.28)$ \\
\hline 25 & People are afraid of someone w/ADHD & 0.718 & $2.60(0.68)$ \\
\hline 16 & Most people are uncomfortable around someone w/ADHD & 0.696 & $2.74(0.65)$ \\
\hline 14 & Others grow distant & 0.683 & $2.70(0.65)$ \\
\hline 12 & Rejected when others find out & 0.639 & $2.66(0.66)$ \\
\hline 26 & Others look for character flaws & 0.626 & $2.62(0.68)$ \\
\hline 22 & Lose friends when they tell others & 0.612 & $2.68(0.65)$ \\
\hline 10 & Most people think those W/ADHD are damaged & 0.574 & $2.75(0.69)$ \\
\hline 20 & People don't want someone w/ADHD around their children & 0.540 & $2.99(0.68)$ \\
\hline 21 & Some people act as though it's the person's fault that they have ADHD & 0.494 & $2.77(0.67)$ \\
\hline \multicolumn{3}{|c|}{ Negative self image } & $23.42(4.26)$ \\
\hline 11 & Feel that they are bad & 0.681 & $2.55(0.65)$ \\
\hline 6 & Feel they aren't as good as others & 0.659 & $2.68(0.66)$ \\
\hline 3 & Risky to tell others & 0.650 & $2.66(0.67)$ \\
\hline 2 & Attitudes make people with ADHD feel worse about themselves & 0.625 & $2.72(0.65)$ \\
\hline 8 & Feel damaged & 0.622 & $2.78(0.64)$ \\
\hline 1 & Feel guilty about having ADHD & 0.616 & $2.23(0.68)$ \\
\hline 9 & Feel set apart and isolated & 0.593 & $2.58(0.72)$ \\
\hline 7 & Treated like outcasts & 0.575 & $2.68(0.69)$ \\
\hline 4 & Lose their jobs because of ADHD & 0.509 & $2.55(0.75)$ \\
\hline \multicolumn{3}{|c|}{ Disclosure concerns } & $22.98(3.83)$ \\
\hline 17 & Worry that others may judge them & 0.737 & $2.87(0.64)$ \\
\hline 15 & Worry about others discriminating & 0.709 & $2.88(0.61)$ \\
\hline 18 & Regret telling people & 0.667 & $2.79(0.65)$ \\
\hline 19 & Feel it was a mistake to tell others & 0.625 & $2.78(0.63)$ \\
\hline 13 & Careful about who to tell & 0.593 & $2.97(0.65)$ \\
\hline 23 & Told others to keep it a secret & 0.591 & $2.80(0.64)$ \\
\hline 5 & Work hard to keep it a secret & 0.536 & $2.96(0.66)$ \\
\hline 24 & Good points tend to be ignored & 0.519 & $2.94(0.68)$ \\
\hline
\end{tabular}

ADHD: attention-deficit/hyperactivity disorder, SD: standard deviation, w: with

using participants' sociodemographic characteristics or diagnosis status of the self, family, relatives, or friends (Table 3).

\section{ASQ scores according to the source of mental health knowledge}

We were interested in the participants' sources of information for mental health and how it affected their stigma towards ADHD. Individuals who acquired most of their information about mental health from the internet demonstrated significantly higher ASQ scores compared to individuals who did not gather information from the internet (Table 4). There were no differences in the ASQ scores from participants who used other sources to obtain information on mental health.

\section{DISCUSSION}

The current study investigated the psychometric proper- ties of the Korean translation of the ASQ. ${ }^{27)}$ The results indicate that the Korean version of the ASQ can be used to measure the stigma towards ADHD in South Korea.

First, we examined the internal consistency of the 26 items in the Korean version of the ASQ. Conventionally, a coefficient alpha of $0.60,0.70$, or 0.80 is considered 'lenient,' 'adequate,' or 'good' scale, respectively. ${ }^{31)}$ The Cronbach's alpha of the 26 items in our study was 0.94 , which indicated excellent internal consistency. Next, exploratory factor analysis was used to reveal the underlying factors. Consistent with the original version of the $\mathrm{ASQ},{ }^{27)}$ three factors were suggested: Disclosure Concerns, Negative Self-Image, and Concern with Public Attitudes. However, some items were endorsed to other factors when compared to the original version of the ASQ. ${ }^{27)}$ Items 3 and 7 were originally included in Disclosure Concerns and items 4,15 , and 24 were originally part of Concern with Public Attitude.

ASQ scores were compared according to the sociodemo- 
Table 3. Comparison of ADHD Stigma Questionnaire scores according to participants' characteristics

\begin{tabular}{|c|c|c|c|}
\hline Characteristics & Mean (SD) & $t / F$ & $\mathrm{p}$ \\
\hline Sex & & 0.54 & 0.59 \\
\hline Male $(n=313)$ & $71.14(11.85)$ & & \\
\hline Female $(n=319)$ & $70.67(10.08)$ & & \\
\hline Age group & & 0.20 & 0.90 \\
\hline $20-29(n=141)$ & $70.64(12.28)$ & & \\
\hline $30-39(n=152)$ & $71.22(10.71)$ & & \\
\hline $40-49(n=183)$ & $70.52(11.16)$ & & \\
\hline $50-59(n=156)$ & $71.27(9.83)$ & & \\
\hline Religion & & 1.03 & 0.30 \\
\hline Yes $(n=310)$ & $70.44(10.83)$ & & \\
\hline No $(n=322)$ & $71.34(11.13)$ & & \\
\hline Family income (won) & & 0.75 & 0.52 \\
\hline$<2000000(n=70)$ & $70.10(11.90)$ & & \\
\hline $2000000-4000000(n=211)$ & $70.20(11.81)$ & & \\
\hline $4000000-6000000(n=205)$ & $71.39(9.86)$ & & \\
\hline$\geq 6000000(n=146)$ & $71.61(10.98)$ & & \\
\hline Educational years & & 0.16 & 0.85 \\
\hline$\leq 12$ & $117.90(27.53)$ & & \\
\hline $13-15$ & $118.88(14.52)$ & & \\
\hline$\geq 16$ & $119.15(18.75)$ & & \\
\hline Any mental disorder of the participant & & 1.12 & 0.26 \\
\hline Yes $(n=48)$ & $72.60(13.02)$ & & \\
\hline No $(n=584)$ & $70.76(10.80)$ & & \\
\hline ADHD diagnosis of family/relative/friends & & 0.75 & 0.45 \\
\hline Yes $(n=80)$ & $70.04(11.08)$ & & \\
\hline No $(n=552)$ & $71.03(10.97)$ & & \\
\hline Any mental disorder of family/relative/friends & & 1.08 & 0.28 \\
\hline Yes $(n=233)$ & $71.52(11.45)$ & & \\
\hline No $(n=399)$ & 70.54 (10.70) & & \\
\hline
\end{tabular}

ADHD: attention-deficit/hyperactivity disorder, SD: standard deviation

Table 4. Comparison of ADHD Stigma Questionnaire scores according to source of mental health knowledge

\begin{tabular}{|c|c|c|c|}
\hline & Mean (SD) & $\dagger$ & $\mathrm{p}$ \\
\hline TV, newspaper & & 1.50 & 0.13 \\
\hline Yes $(n=428)$ & $71.35(10.83)$ & & \\
\hline No $(n=204)$ & $69.95(11.26)$ & & \\
\hline Internet search & & 2.53 & 0.01 \\
\hline Yes $(n=459)$ & $71.58(11.10)$ & & \\
\hline No $(n=173)$ & $69.11(10.49)$ & & \\
\hline Book & & 0.28 & 0.78 \\
\hline Yes $(n=252)$ & $71.05(10.02)$ & & \\
\hline No $(n=380)$ & $70.80(11.59)$ & & \\
\hline Mental health education class & & 0.57 & 0.57 \\
\hline Yes $(n=110)$ & $71.45(9.35)$ & & \\
\hline No $(n=522)$ & $70.79(11.30)$ & & \\
\hline Family, friends, and acquaintance & & -0.62 & 0.54 \\
\hline Yes $(n=202)$ & $70.51(10.72)$ & & \\
\hline No $(n=430)$ & $71.09(11.11)$ & & \\
\hline
\end{tabular}

ADHD: attention-deficit/hyperactivity disorder, SD: standard deviation 
graphic variables of the participants. However, there were no significant differences between ASQ scores based on the variables that we examined. This suggests that stigma against ADHD exists in all sociodemographic groups. This is similar to the findings of previous studies in which stigma towards individuals with ADHD appears in childhood ${ }^{32)}$ and continues through adulthood." Also, a previous study ${ }^{33)}$ found no differences in teachers' ASQ scores when examined for age, gender, and ethnicity.

Lastly, we examined the sources of participants' information on mental disorders and how that affects their stigma towards ADHD. Individuals who acquired information about mental health from the internet showed higher ASQ scores compared to those who used other sources of information. This suggests that policy makers and researchers in South Korea should focus on how mental health-related information is portrayed on the internet. When it is used properly, social media could be very effective in reducing stigma towards individuals with mental disorders. ${ }^{34)}$ Additionally, a recent review study ${ }^{35)}$ states that web-based mental health literacy interventions were effective in reducing stigma towards mental illness. However, this result was based on a selfreport measure, therefore more studies need to be conducted on this subject.

The current study has several limitations. First, we did not use a nationally representative sample. Therefore, the representativeness of our results is limited. Future studies should investigate the psychometric properties of the Korean version of the ASQ using a nationally representative sample. Another limitation is that we utilized a cross-sectional design. Therefore, we could not examine the causal relationships between stigma and the source of information about mental health.

\section{CONCLUSION}

To our knowledge, this is the first study to investigate the psychometric properties of an assessment tool measuring stigma against ADHD in South Korea. The Korean translation of the ASQ has acceptable psychometric properties among Korean adults. Additionally, the study results suggest that inaccurate information from the internet could increase the stigma surrounding ADHD. Future studies should focus on the effectiveness of providing an intervention to reduce stigma towards ADHD by removing misleading information and providing proper information about ADHD on the internet.

\section{Acknowledgments}

This work was supported by an intramural grant (No. R2018-A) from the National Center for Mental Health, Republic of Korea.

\section{Conflicts of Interest}

The authors have no financial conflicts of interest.

\section{REFERENCES}

1) American Psychiatric Association. Diagnostis and statistical manual of mental disorders. Washington, DC: American Psychiatric Association;2013.

2) Polanczyk G, de Lima MS, Horta BL, Biederman J, Rohde LA. The worldwide prevalence of ADHD: a systematic review and metaregression analysis. Am J Psychiatry 2007;164:942-948.

3) Lara C, Fayyad J, de Graaf R, Kessler RC, Aguilar-Gaxiola S, Angermeyer M, et al. Childhood predictors of adult ADHD: results from the WHO World Mental Health (WMH) survey initiative. Biol Psychiatry 2009;65:46-54.

4) Fabrega $\mathrm{H}$ Jr. Psychiatric stigma in the classical and medieval period: a review of the literature. Compr Psychiatry 1990;31:289-306.

5) Goffman E. Stigma: notes on the management of spoiled identity. Englewood Cliffs, NJ: Prentice Hall;1963.

6) Canu WH, Newman ML, Morrow TL, Pope DL. Social appraisal of adult ADHD: stigma and influences of the beholder's Big Five personality traits. J Atten Disord 2008;11:700-710.

7) Mueller AK, Fuermaier AB, Koerts J, Tucha L. Stigma in attention deficit hyperactivity disorder. Atten Defic Hyperact Disord 2012;4: 101-114.

8) Corrigan PW, Kleinlein P. The impact of mental illness stigma. On the stigma of mental illness: practical strategies for research and social change. Washington, DC: American Psychological Association;2005. p.11-44.

9) Corrigan PW, Shapiro JR. Measuring the impact of programs that challenge the public stigma of mental illness. Clin Psychol Rev 2010;30:907-922.

10) Corrigan PW, Markowitz FE, Watson AC. Structural levels of mental illness stigma and discrimination. Schizophr Bull 2004;30:481491.

11) Thornicroft G, Rose D, Kassam A. Discrimination in health care against people with mental illness. Int Rev Psychiatry 2007;19: 113-122.

12) Hoza B, Mrug S, Gerdes AC, Hinshaw SP, Bukowski WM, Gold JA, et al. What aspects of peer relationships are impaired in children with attention-deficit/hyperactivity disorder? J Consult Clin Psychol 2005;73:411-423.

13) Tuchman G. EES presidential address, 1995-invisible differences: on the management of children inpostindustrial society. Sociol Forum 1996;11:3-23.

14) Norvilitis JM, Scime M, Lee JS. Courtesy stigma in mothers of children with attention deficit/hyperactivity disorder: a preliminary investigation. J Atten Disord 2002;6:61-68.

15) Hinshaw SP, Stier A. Stigma as related to mental disorders. Annu Rev Clin Psychol 2008;4:367-393.

16) Brohan E, Slade M, Clement S, Thornicroft G. Experiences of mental illness stigma, prejudice and discrimination: a review of measures. BMC Health Serv Res 2010;10:80.

17) Phelan JC, Link BG, Stueve A, Pescosolido BA. Public conceptions of mental illness in 1950 and 1996: what is mental illness and is it to be feared? J Health Soc Behav 2000;41:188-207.

18) Angermeyer MC, Dietrich S. Public beliefs about and attitudes towards people with mental illness: a review of population studies. Acta Psychiatr Scand 2006;113:163-179.

19) Wood L, Birtel M, Alsawy S, Pyle M, Morrison A. Public perceptions of stigma towards people with schizophrenia, depression, and 
anxiety. Psychiatry Res 2014;220:604-608.

20) Thornicroft G, Brohan E, Rose D, Sartorius N, Leese M; INDIGO Study Group. Global pattern of experienced and anticipated discrimination against people with schizophrenia: a cross-sectional survey. Lancet 2009;373:408-415.

21) Wang J, Lai D. The relationship between mental health literacy, personal contacts and personal stigma against depression. J Affect Disord 2008;110:191-196.

22) Hawke LD, Parikh SV, Michalak EE. Stigma and bipolar disorder: a review of the literature. J Affect Disord 2013;150:181-191.

23) Sadler MS, Meagor EL, Kaye KE. Stereotypes of mental disorders differ in competence and warmth. Soc Sci Med 2012;74:915-922.

24) Kramer EJ, Kwong K, Lee E, Chung H. Cultural factors influencing the mental health of Asian Americans. West J Med 2002;176: 227-231.

25) Lipson JG, Dibble SL, Minarik PA. Culture \& nursing care: a pocket guide. San Francisco, CA: UCSF Nursing Press;1996.

26) Park JI, Jeon M. The stigma of mental illness in Korea. J Korean Neuropsychiatr Assoc 2016;55:299-309.

27) Kellison I, Bussing R, Bell L, Garvan C. Assessment of stigma associated with attention deficit hyperactivity disorder: psychometric evaluation of the ADHD stigma questionnaire. Psychiatry Res 2010;178:363-369.
28) Sieff E. Media frames of mental illnesses: the potential impact of negative frames. J Ment Health 2003;12:259-269.

29) Klin A, Lemish D. Mental disorders stigma in the media: review of studies on production, content, and influences. J Health Commun 2008:13:434-449.

30) Berger BE, Ferrans CE, Lashley FR. Measuring stigma in people with HIV: psychometric assessment of the HIV stigma scale. Res Nurs Health 2001;24:518-529.

31) Litwin MS. How to assess and interpret survey psychometrics. Thousand Oaks, CA: Sage Publications;2002.

32) Coleman D, Walker JS, Lee J, Friesen BJ, Squire PN. Children's beliefs about causes of childhood depression and ADHD: a study of stigmatization. Psychiatr Serv 2009;60:950-957.

33) Bell L, Long S, Garvan C, Bussing R. The impact of teacher credentials on ADHD stigma perceptions. Psychol Sch 2011;48:184-197.

34) Betton V, Borschmann R, Docherty M, Coleman S, Brown M, Henderson $\mathrm{C}$. The role of social media in reducing stigma and discrimination. Br J Psychiatry 2015;206:443-444.

35) Brijnath B, Protheroe J, Mahtani KR, Antoniades J. Do web-based mental health literacy interventions improve the mental health literacy of adult consumers? results from a systematic review. J Med Internet Res 2016;18:e165. 